\section{High-Resolution Multi-Contrast MRI of the Carotid Artery Wall for Evaluation of Atherosclerotic Plaques}

MRI can be used for noninvasive detection and characterization of atherosclerotic plaques in extracranial carotid arteries (Yuan et al., 2001a). MRI provides information about plaque geometry, distribution, and tissue composition. Once atherosclerotic disease is detected, follow-up scans may be helpful in the evaluation of disease progression and effect of treatment.

This unit presents a protocol for multi-contrast, high-resolution imaging of the wall of extracranial carotid arteries. Identification of basic plaque components (fibrous tissue, lipid-rich necrotic core, calcification) and incidental changes (a rupture or hemorrhage) can be accomplished by comparison of black-blood images with basic contrast weightings- $T_{1}$-weighted, $T_{2}$-weighted, and proton density (PD)-weighted-in conjunction with bright-blood images obtained using time-of-flight MR angiography (TOF MRA).

This Basic Protocol summarizes the authors' experience at $1.5 \mathrm{~T}$ with the use of several models of GE scanners (Signa Echospeed, LX, and CVi) and a Philips Gyroscan NT scanner. Use of the protocol with different field strengths may require special tests in order to optimize sequence parameters because of changes in relaxation times and the signal-to-noise ratio (SNR).

The entire protocol consists of seven imaging sequences. The first two sequences are used to locate the bilateral carotid bifurcations. Sequence 3 (black-blood MRA of the carotid bifurcation) allows one to precisely determine the position of the bifurcation, which is then used as a landmark for all subsequent transverse sequences 4 to 7 . The side of the bifurcation chosen for this purpose is referred to below as the index side. The index side is recorded for use in follow-up examinations, if indicated. Sequence 3 is also helpful to visualize plaque distribution along the artery. If necessary, this sequence can be repeated for the contralateral side. Four transverse scans (sequences 4 to 7) provide black-blood images with basic contrast weightings ( $T_{1}$-weighted, PD-weighted, and $T_{2}$-weighted) and bright-blood (3-D TOF) images, which are necessary for plaque characterization. In order to facilitate further comparisons, coverage of sequences 4 through 7 is prescribed so that slices obtained from different sequences are positioned at the same distance from the carotid bifurcation of the index side. The Basic Protocol takes $\sim 30$ to 40 min depending on coverage (number of slices). The entire examination, including patient setup, will take $\sim 1 \mathrm{hr}$.

Table A1.4.1 lists the hardware necessary to perform the procedure, along with the appropriate parameters. The available gradient strength will depend on the scanner, and the echo times given in other tables below may be varied accordingly (the smaller the gradient strength, the longer the echo time for a particular scan).

NOTE: Be sure that technologists and nurses have immediate access to any emergency equipment that may be relevant to a given study, or that may be needed for a particular patient, such as crash carts or oxygen.

\section{Set up patient and equipment}

1. Interview (screen) the patient to ensure that he or she has no contraindications such as cardiac pacemakers or other implants containing ferromagnetic materials that may be problematic for patient safety or good image acquisition. Also be sure to find out

Contributed by Vasily L. Yarnykh and Chun Yuan

Current Protocols in Magnetic Resonance Imaging (2003) A1.4.1-A1.4.17

Copyright () 2003 by John Wiley \& Sons, Inc.
UNIT A1.4

BASIC

PROTOCOL

Intracranial Arterial Disease

A1.4.1

Supplement 11 
Table A1.4.1 Equipment Parameters for High-Resolution Multicontrast MRI of the Carotid Artery Wall

\begin{tabular}{ll}
\hline Coil type & $\begin{array}{l}\text { Phased-array bilateral surface carotid coil } \\
\text { (Fig. A1.4.1) }\end{array}$ \\
Gradient coil strength & 25 mT/m (or whatever the system permits) \\
Cardiac gating & No \\
Peripheral gating & Yes (can be omitted if a double \\
& inversion-recovery, DIR, method is used \\
& for blood suppression in sequences 3-6) \\
Respiratory gating & No \\
Respirator & If required by patient \\
Oxygen & If required by patient \\
Motion cushions & Useful \\
Use of contrast agents & No \\
\hline
\end{tabular}

if the patient has any health conditions that may require the presence of special emergency equipment during the scanning procedure, or necessitate any other precautions.

Generally, standard screening forms (see APPENDIX 1) are used for all patients scanned in a magnetic resonance system.

The presence of any ferromagnetic metals may be a health hazard to the patient when he or she is inside the magnet, and will also affect the imaging. If in doubt as to the exact composition of the items, it is best to exclude patients with any metal implants; see Shellock (2001) for discussion of what implants may be safely scanned using magnetic resonance.

Patients may be accompanied into the magnet room by a friend or family member, who can sit in the room during the scan and comfort the patient as needed. This companion must be screened as well to ensure the absence of loose metal objects on the body or clothing, as well as other items as described above.

2. If the procedure is a research protocol, have the patient sign any necessary consent forms.

3. Have the patient remove all jewelry and change into a gown to eliminate any metal that might be found in clothing.

4. Have the patient wash off any mascara and other makeup to avoid local tissue heating and image artifacts.

5. Inform the patient of what will occur during the procedure, what he or she will experience while in the magnet, and how to behave, including the following:

a. If earplugs, earphones, or headphones are used to protect the ears from the loud sounds produced by the gradients, the patient will be asked to wear one of these devices, but will be able to communicate with you at any time during the imaging.

b. The patient may be given a safety squeeze-bulb or similar equipment to request assistance at any time (demonstrate how this works).

c. For good results, the patient should not talk, and should avoid or minimize swallowing or other movement during each scan-i.e., as long as the banging sounds continue. Between scans, talking and swallowing are allowed in most cases, but should be avoided when comparative positional studies are being performed; the patient will be informed when this is the case.

d. Nevertheless, the patient may call out at any time if he or she feels it necessary. 


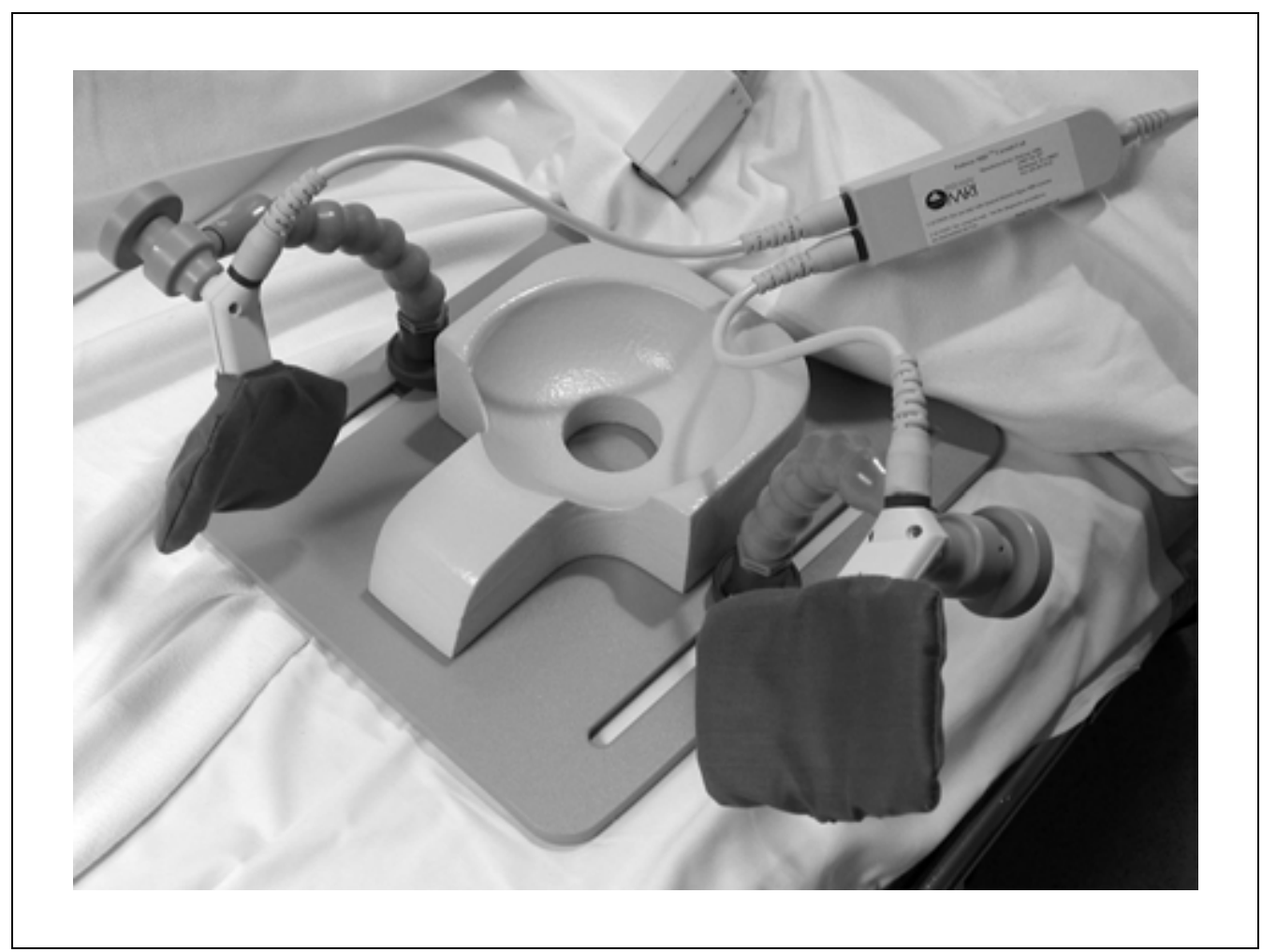

Figure A1.4.1 Photo of the bilateral phased-array carotid coil with head holder (Pathway Medical).

6. Set up the carotid phased-array coil with head holder (Fig. A1.4.1) on the moving table at approximately the same position as for a standard head coil. Have the patient mount onto the table in a supine position with head placed on the head holder. After the patient lies down, attach a peripheral gating lead to the patient's finger and check the appearance of the appropriate triggering signal according to manufacturer's guidelines.

The need for peripheral gating depends on the particular setup of sequences 3 to 6. Gating improves quality of blood suppression in sequences 5 and 6, if inflow saturation pulses are employed. However, gating can be omitted if a double inversion-recovery (DIR) method is used for blood suppression in sequences 3 through 6, and if the software of the scanner allows non-gated acquisition with DIR. See annotations in corresponding sequences for details.

7. Place bilateral receiver panels of the carotid phased-array coil (Fig. A1.4.1) on the left and right sides of the patient's neck. Fix the coil panels using setscrews. If necessary, adjust the coil panels for the comfort of the patient.

Generally, the head of the patient is fixed so that the head is horizontal (not tilted) and the neck and head lie along the axis of the table.

If a special carotid phased-array coil is unavailable, other small surface coils (like a coil for temporomandibular joints scanning, TMJ coil) can be used if they can provide sufficient signal-to-noise ratio.

8. If needed, place a pillow or other support under the knees to make the patient more comfortable.

9. Use the centering light to position the patient at the angle of the mandible and put him or her into the center of the magnet. 
Table A1.4.2 Primary Clinical Imaging Parameters for Sequence 1 (Pilot Scan)

\begin{tabular}{ll}
\hline Patient position & Supine \\
Scan type & 2-D gradient echo \\
Imaging plane (orientation) & $\begin{array}{l}\text { Sagittal/coronal/transverse (many } \\
\text { scanners are capable of triple plane } \\
\text { orthogonal scouts) }\end{array}$ \\
& Laser light centered on edge of jaw \\
Central slice or volume center & As short as possible $(\sim 2-6 \mathrm{msec})$ \\
Echo time $\left(T_{\mathrm{E}}\right)$ & As short as possible $(\sim 10-15 \mathrm{msec})$ \\
Repeat time $\left(T_{\mathrm{R}}\right)$ & $15^{\circ}$ \\
Flip angle $(\mathrm{FA})$ & $250 \mathrm{~mm}, 250 \mathrm{~mm}$ \\
Fields of view $\left(\mathrm{FOV}_{x}, \mathrm{FOV}_{y}\right)$ & $0.98 \mathrm{~mm}, 1.95 \mathrm{~mm}$ \\
Resolution $(\Delta x, \Delta y)$ & 256,128 \\
Number of data points collected $\left(N_{\mathrm{x}}, N_{\mathrm{y}}\right)$ & 256,256 \\
Display matrix $\left(D_{\mathrm{x}}, D_{\mathrm{y}}\right)$ & $5-7 \mathrm{~mm}$ \\
Slice thickness $(\Delta z)$ & $3-22(1-12$ sagittal, $1-5$ coronal, $1-5$ \\
Number of slices & transverse $)$ \\
Slice gap & $0-2 \mathrm{~mm}$ \\
Number of acquisitions $\left(N_{\mathrm{acq}}\right)$ & 1 \\
Scan time & $\sim 5-40 \mathrm{sec}$ \\
\hline
\end{tabular}

Table A1.4.3 Primary Clinical Imaging Parameters for Sequence 2 (2-D TOF)

\begin{tabular}{ll}
\hline Patient position & Supine \\
Scan type & 2-D spoiled gradient echo \\
Imaging plane (orientation) & Transverse \\
Variable bandwidth & Yes \\
Central slice or volume center & Close to the carotid bifurcations \\
Echo time $\left(T_{\mathrm{E}}\right)$ & As short as possible $(<5 \mathrm{msec})$ \\
Receiver bandwidth $(\mathrm{RBW})$ & $\pm 16 \mathrm{kHz}$ or close \\
Repeat time $\left(T_{\mathrm{R}}\right)$ & As short as possible $(\sim 10-20 \mathrm{msec})$ \\
Flip angle $(\mathrm{FA})$ & $30^{\circ}$ \\
Fields of view $\left(\mathrm{FOV}_{x}, \mathrm{FOV}_{y}\right)$ & $160 \mathrm{~mm}, 120 \mathrm{~mm}$ \\
Resolution $(\Delta x, \Delta y)$ & $0.63 \mathrm{~mm}, 0.94 \mathrm{~mm}$ \\
Number of data points collected $\left(N_{\mathrm{x}}, N_{\mathrm{y}}\right)$ & 256,128 \\
Display matrix $\left(D_{\mathrm{x}}, D_{\mathrm{y}}\right)$ & 256,256 \\
Slice thickness $(\Delta \mathrm{z})$ & $2-3 \mathrm{~mm}$ \\
Number of slices & $30-50$ or as many as needed to \\
& visualize carotid bifurcations \\
Slice gap & 0 mm \\
Number of acquisitions $\left(N_{\mathrm{acq}}\right)$ & 1 \\
Read direction & Left-right \\
Slice locations & Carotid bifurcation \\
Flow compensation & Yes \\
Saturation pulses & Yes; superior to saturate veins \\
Slice series & Sequential, ascending \\
Scan time & $\sim 0.5-2$ min \\
\hline
\end{tabular}


Table A1.4.4 Primary Clinical Imaging Parameters for Sequence 3 (Oblique 2-D Black-Blood MRA) ${ }^{a}$

\begin{tabular}{|c|c|}
\hline Patient position & Supine \\
\hline Scan type & $\begin{array}{l}\text { 2-D FSE (fast spin echo) with DIR } \\
\text { preparation }\end{array}$ \\
\hline Imaging plane (orientation) & Oblique-sagittal \\
\hline Variable bandwidth & Yes \\
\hline Central slice or volume center & $\begin{array}{l}\text { Along line connecting internal and } \\
\text { external carotid arteries }\end{array}$ \\
\hline Echo time $\left(T_{\mathrm{E}}\right)$ & As short as possible $(\sim 7-10 \mathrm{msec})$ \\
\hline Receiver bandwidth (RBW) & $31 \mathrm{kHz}$ or close \\
\hline Echo train length (ETL) & 16 \\
\hline Repeat time $\left(T_{\mathrm{R}}\right)$ & $2 \mathrm{R}$-to-R intervals ${ }^{a}$ or $1600 \mathrm{msec}^{b}$ \\
\hline Inversion time $\left(T_{\mathrm{I}}\right)$ & $550 \mathrm{msec}^{b}$ \\
\hline Fields of view $\left(\mathrm{FOV}_{x}, \mathrm{FOV}_{y}\right)$ & $160 \mathrm{~mm}, 160 \mathrm{~mm}$ \\
\hline Resolution $(\Delta x, \Delta y)$ & $0.63 \mathrm{~mm}, 0.63 \mathrm{~mm}$ \\
\hline Number of data points collected $\left(N_{\mathrm{x}}, N_{\mathrm{y}}\right)$ & 256,256 \\
\hline Display matrix $\left(D_{\mathrm{x}}, D_{\mathrm{y}}\right)$ & 512,512 \\
\hline Slice thickness $(\Delta \mathrm{z})$ & $2 \mathrm{~mm}$ \\
\hline Number of slices & $5-7$ \\
\hline Slice gap & $0 \mathrm{~mm}$ \\
\hline Number of acquisitions $\left(N_{\text {acq }}\right)$ & 2 \\
\hline Read direction & Superior-inferior \\
\hline Slice locations & Carotid bifurcation \\
\hline Flow compensation & No \\
\hline ZIP 512 & Yes \\
\hline Saturation pulses & No \\
\hline Fat suppression & Yes \\
\hline Slice series & Sequential \\
\hline Scan time & $\sim 50-60 \mathrm{sec} /$ slice \\
\hline
\end{tabular}

Once this step has been performed, so long as the patient does not move on the table, the table itself can be moved and then returned to the same position as before without jeopardizing the positioning of one scan relative to another.

10. If the patient is unable to hold still, provide an appropriate sedative.

\section{Sequence 1: Rapid three-plane positioning pilot}

11. Run the system's pilot (or scout) scan to ensure correct location of the neck in three dimensions, using the imaging sequence given in Table A1.4.2 or similar parameters.

This sequence usually consists of three orthogonal planes to allow localization. The images are often also used later to determine where to place the saturation pulses and to set up total coverage of the volume of interest.

If allowed by a scanner, the authors recommend using a group of slices for each plane, i.e., 8 to 12 sagittal, 3 to 5 coronal, and 3 to 5 transverse slices. Lateral sagittal images can be used to locate carotid bifurcations. If the bifurcations are visible on scout images, a fewer number of slices can be used for the next sequence (2-D TOF MRA). 
Table A1.4.5 Alternate Imaging Parameters for Sequence 3 (Oblique 2-D Black-Blood MRA) with the Use of a Multislice DIR Method ${ }^{a}$

\begin{tabular}{|c|c|}
\hline Patient position & Supine \\
\hline Scan type & 2-D FSE with DIR preparation \\
\hline Imaging plane (orientation) & Oblique-sagittal \\
\hline Variable bandwidth & Yes \\
\hline Pulse sequence database (PSD) & "fse-m"a \\
\hline Central slice or volume center & $\begin{array}{l}\text { Along line connecting internal and } \\
\text { external carotid arteries }\end{array}$ \\
\hline Echo time $\left(T_{\mathrm{E}}\right)$ & As short as possible $(\sim 7-10 \mathrm{msec})$ \\
\hline Receiver bandwidth (RBW) & $\pm 31 \mathrm{kHz}$ or close \\
\hline Echo train length (ETL) & 16 \\
\hline Repeat time $\left(T_{\mathrm{R}}\right)$ & $1800 \mathrm{msec}^{b}$ \\
\hline Inversion time $\left(T_{\mathrm{I}}\right)$ & $270 \mathrm{msec}^{b}$ \\
\hline Fields of view $\left(\mathrm{FOV}_{x}, \mathrm{FOV}_{y}\right)$ & $160 \mathrm{~mm}, 160 \mathrm{~mm}$ \\
\hline Resolution $(\Delta x, \Delta y)$ & $0.63 \mathrm{~mm}, 0.63 \mathrm{~mm}$ \\
\hline Number of data points collected $\left(N_{\mathrm{x}}, N_{\mathrm{y}}\right)$ & 256,256 \\
\hline Display matrix $\left(D_{\mathrm{x}}, D_{\mathrm{y}}\right)$ & 512,512 \\
\hline Slice thickness $(\Delta z)$ & $2 \mathrm{~mm}$ \\
\hline Number of slices & $6^{b}$ \\
\hline Slice gap & $0 \mathrm{~mm}$ \\
\hline Number of acquisitions $\left(N_{\text {acq }}\right)$ & 2 \\
\hline Number of repetitions & 2 \\
\hline Read direction & Superior-inferior \\
\hline Slice locations & Carotid bifurcation \\
\hline Flow compensation & No \\
\hline ZIP 512 & Yes \\
\hline Saturation pulses & No \\
\hline Fat suppression & Yes \\
\hline Slice series & Interleaved \\
\hline Scan time & $\sim 2 \mathrm{~min} / 6$ slices \\
\hline
\end{tabular}

For older models of MR scanners where three-plane scans may be unavailable, use a rapid gradient-echo scan in a sagittal plane. Prescribe 12 to 16 slices to ensure coverage of the neck in the left-right direction.

\section{Sequence 2: 2-D time-of-flight MRA}

12. Set up the imaging parameters according to Table A1.4.3.

13. Use the scout images obtained earlier to locate the carotid arteries and ensure coverage of the region of interest. Prescribe slices graphically to cover long enough segments of the carotid arteries, including both bifurcations. Use the pilot images to set up a superior saturation band for suppression of venous flow signal.

14. Start scan and ensure the appearance of both left and right bifurcations on the obtained images. If, occasionally, bifurcations are not found, repeat this scan with superior or inferior shift of a slice group (slab) until bifurcations are found. 

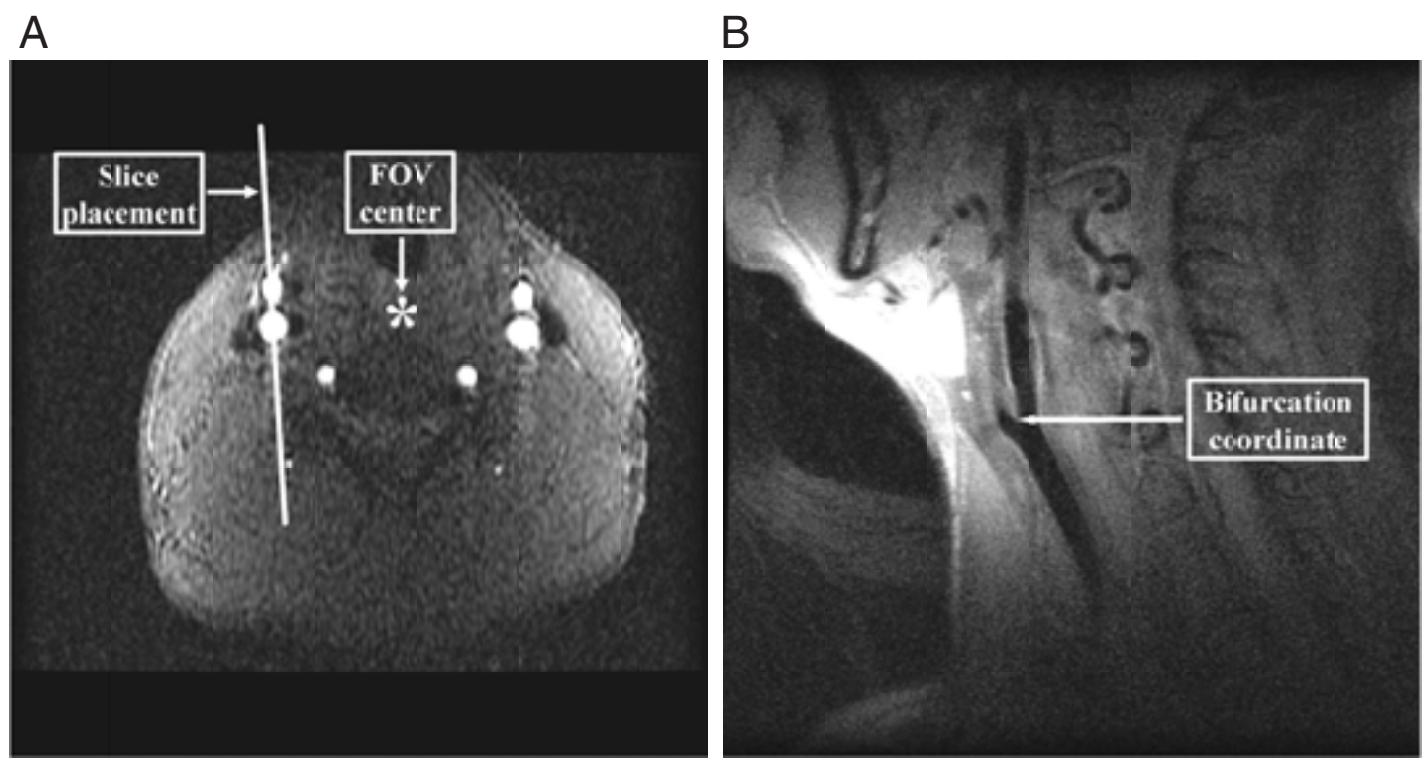

Figure A1.4.2 Setting scan geometry. (A) Position of slices for sequence 3 (oblique sagittal black-blood MRA) using transverse 2-D TOF MRA (sequence 2) as a localizer. 2-D TOF MRA image is also used to determine coordinates of the FOV (field of view) center (asterisk) for all subsequent transverse sequences. (B) Finding an inferior-superior coordinate of the bifurcation (arrow) on the oblique black-blood image obtained in sequence 3.

This sequence uses sequential slice acquisition. On most scanners, acquired slices are reconstructed immediately, and the operator may see recently obtained images. When both left and right bifurcations are passed and 5 to 10 images are obtained above the bifurcations, acquisition can be stopped to save scan time.

\section{Sequence 3: Oblique sagittal 2-D black-blood MRA of the carotid bifurcation}

15. Set up parameters according to Table A1.4.4.

This sequence uses a double inversion-recovery (DIR) method (Edelman et al., 1991) to obtain images with heavily suppressed blood signal. In the specifications of the scanners, DIR is often referred to as "blood suppression" or "black blood" option. Sequence 3 can be run in either non-gated or gated mode. In most commercially available scanner software versions, gating is a default option for black-blood imaging. In the authors' experience, gating does not improve blood suppression or image quality for this sequence, and therefore a custom-designed non-gated sequence is used. In patients with arrhythmias or an abnormally fast or slow heart rate, non-gated acquisition is preferable. In such situations, a modified (non-gated) pulse sequence or gating simulator should be used.

Scan time for sequence 3 can be considerably reduced using a new multi-slice DIR technique (Yarnykh and Yuan, 2003). If a multi-slice DIR pulse sequence is available, set up parameters according to Table A1.4.5.

16. Review the images obtained in sequence 2 . Find the carotid bifurcation on the index side. Locate the first slice above the bifurcation, where internal and external carotid arteries are seen separately (Fig. A1.4.2A). Use this slice as a locator in the graphic display. Place a slice group (slab) to be imaged along the line connecting the internal and external carotid artery (Fig. A1.4.2A).

17. Instruct the patient to refrain from swallowing during the scan and run the scan.

18. If indicated by a local protocol, repeat steps 16 and 17 for the contralateral side.

The index side can be marked by a physician or chosen during the examination based on the appearance of stenosis on MRA images (sequence 2). Black-blood MRA is also useful for estimation of plaque distribution along the artery. For purposes of surgical planning,

Intracranial Arterial Disease 
A

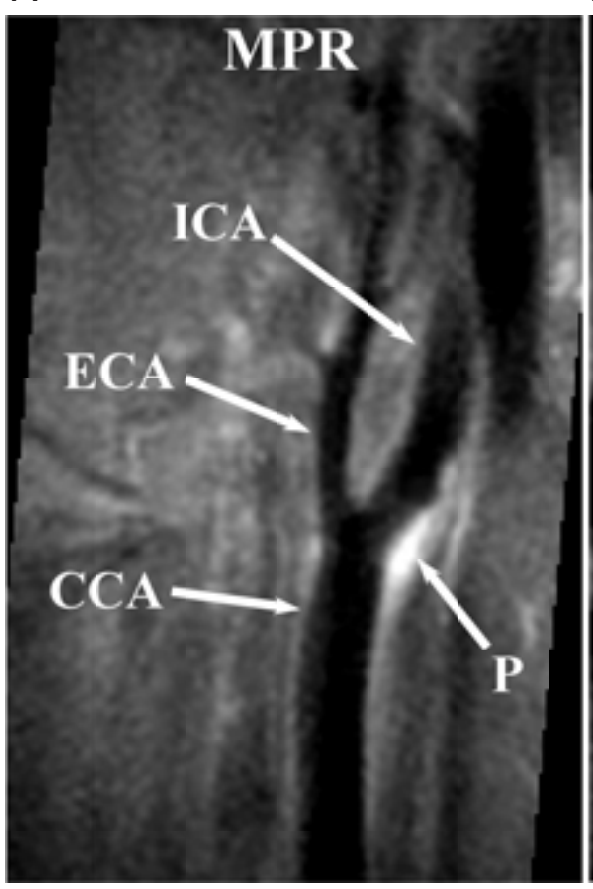

B

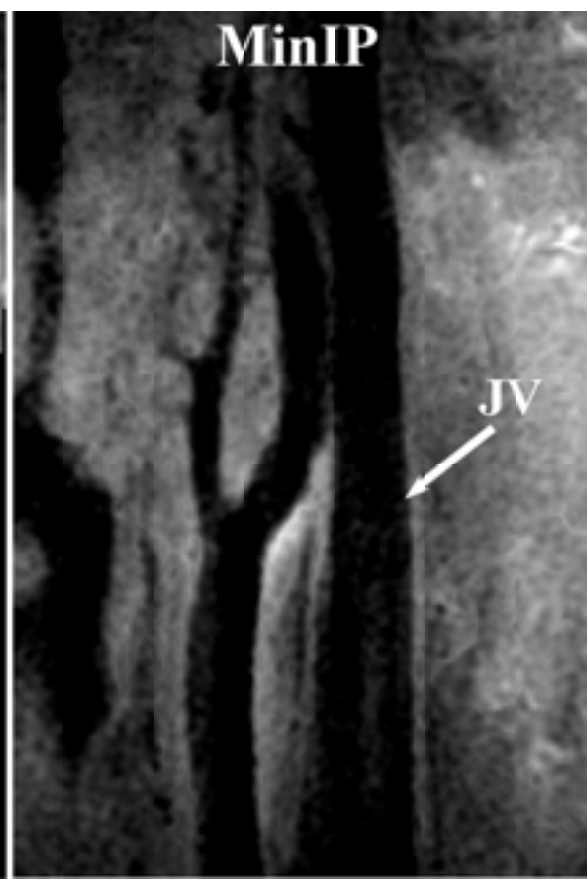

Figure A1.4.3 Oblique sagittal 2-D black-blood MRA images (sequence 3) processed by multiplanar reformation (MPR; A) and minimal-intensity projection (MinIP; B). Images were obtained at the left side from a patient with moderate atherosclerotic disease. Transverse images of this patient are shown in Figure A1.4.4. Abbreviations correspond to common (CCA), internal (ICA), end external (ECA) carotid arteries, jugular vein (JV), and plaque (P). Reformatted view (A) shows a fragment of plaque $(P)$, whereas minimal-intensity projection $(B)$ confirms that the lumen is almost unobstructed.

an additional scan with sequence 3 can be obtained in the oblique plane orthogonal to the plaque surface at the point of maximal thickness. Such a scan can be prescribed after obtaining transverse images (sequences 4 through 7 ).

\section{Image processing and viewing for sequences 2 and 3}

19. Find bilateral carotid bifurcations in images obtained from sequence 2. Find the approximate position of the central point at the line connecting the bifurcations using the cursor (Fig. A1.4.2A). Document "left-right" and "anterior-posterior" coordinates of the central point for future use.

These coordinates will be used to ensure identical positioning of the center of the field of view (FOV) in the next sequences.

20. Review the images obtained from sequence 3 for the index side (Fig. A1.4.2B). If necessary, adjust window level to clearly see a dark lumen of the carotid artery. Zoom images $\sim 2 \times$.

21. Find slice(s) containing the carotid bifurcation (Fig. A1.4.2B). If several slices dissect the bifurcation, find the slice with the lowest (i.e., most inferior) position of the bifurcation using the cursor. Document the inferior-superior coordinate of the bifurcation for future use.

The carotid bifurcation on the index side is used as the internal reference for prescription of transverse multi-contrast images in the next sequences. Use of the above coordinates facilitates registration between images with different contrast weightings. If follow-up 
Table A1.4.6 Primary Clinical Imaging Parameters for Sequence 4 (Transverse $T_{1}$-Weighted Black-Blood FSE) ${ }^{a}$

\begin{tabular}{|c|c|}
\hline Patient position & Supine \\
\hline Scan type & 2-D FSE with DIR preparation \\
\hline Imaging plane (orientation) & Transverse \\
\hline Variable bandwidth & Yes \\
\hline Central slice or volume center & Carotid bifurcation or stenosis \\
\hline Echo time $\left(T_{\mathrm{E}}\right)$ & As short as possible $(\sim 7-11 \mathrm{msec})$ \\
\hline Receiver bandwidth (RBW) & $\begin{array}{l} \pm 21 \mathrm{kHz} \text { or close (or } \pm 31 \mathrm{kHz} \text { if } T_{\mathrm{R}}=2 \\
\mathrm{R} \text {-to-R intervals) }\end{array}$ \\
\hline Echo train length (ETL) & 10 (or 12 if $T_{\mathrm{R}}=2 \mathrm{R}$-to-R intervals) ${ }^{b}$ \\
\hline Repeat time $\left(T_{\mathrm{R}}\right)$ & $\begin{array}{l}800 \mathrm{msec}^{c} \text { or } 1 \mathrm{R} \text {-to- } \mathrm{R} \text { interval }{ }^{a} \text { or } 2 \\
\mathrm{R} \text {-to- } \mathrm{R} \text { intervals }\end{array}$ \\
\hline Inversion time $\left(T_{\mathrm{I}}\right)$ & $330 \mathrm{msec}^{c}$ \\
\hline Fields of view $\left(\mathrm{FOV}_{x}, \mathrm{FOV}_{y}\right)$ & $160 \mathrm{~mm}, 120 \mathrm{~mm}$ \\
\hline Resolution $(\Delta x, \Delta y)$ & $0.63 \mathrm{~mm}, 0.63 \mathrm{~mm}$ \\
\hline Number of data points collected $\left(N_{\mathrm{x}}, N_{\mathrm{y}}\right)$ & 256,192 \\
\hline Display matrix $\left(D_{\mathrm{x}}, D_{\mathrm{y}}\right)$ & 512,512 \\
\hline Slice thickness $(\Delta \mathrm{z})$ & $2 \mathrm{~mm}$ \\
\hline Number of slices & $8-16$ \\
\hline Slice gap & $0 \mathrm{~mm}$ \\
\hline Number of acquisitions $\left(N_{\mathrm{acq}}\right)$ & 2 \\
\hline Read direction & Left-right \\
\hline Slice locations & $\begin{array}{l}\text { Precise position relative to carotid } \\
\text { bifurcation }\end{array}$ \\
\hline Flow compensation & No \\
\hline ZIP 512 & Yes \\
\hline Saturation pulses & No \\
\hline Fat suppression & Yes \\
\hline Slice series & Sequential \\
\hline Scan time & $\sim 30-60 \mathrm{sec} /$ slice \\
\hline \multicolumn{2}{|c|}{$\begin{array}{l}{ }^{a} \text { Depending on the specifications of the scanner, this sequence may require peripheral gating. Alternatively, } \\
\text { the sequence can be run without gating (if allowed by a scanner) or with gating simulator. } \\
{ }^{b} \text { Specific parameter setting for GE scanners, if the sequence is gated to actual patient's heart rate with } T_{\mathrm{R}} \\
=2 \mathrm{R} \text {-to-R intervals. } \\
{ }^{c} T_{\mathrm{R}} \text { for a non-gated sequence. The delay between DIR pulses and an acquisition sequence (if available for } \\
\text { control) should be set to } 330 \mathrm{msec} \text {. }\end{array}$} \\
\hline
\end{tabular}

examinations of a patient are expected, in each examination, transverse images should be obtained at the same distance from the bifurcation on the index side.

22. If indicated, perform multi-planar reformation of images obtained from sequence 3 . Load images into the multi-planar reformation tool. Rotate reformatted plane to clearly visualize the extent of the plaque into the lumen and its relationship to the common, internal and external carotid arteries (Fig. A1.4.3A). If also indicated, prepare a minimal intensity projection ( $\min \mathrm{IP}$ ) for the image subset crossing the carotid arteries (Fig. A1.4.3B).

This step can be done after the examination or during the acquisition of subsequent sequences. A particular algorithm of image processing depends on available software tools and clinical requirements. Generally, multi-planar views may help to estimate the distribution of the plaque along the artery. A minimal intensity projection may allow the physician to estimate the degree of lumen obstruction. However, care must be taken near adjacent jugular veins, which may interfere with the carotid arteries on minimal intensity projections.

Intracranial Arterial Disease

A1.4.9

Supplement 11 
Table A1.4.7 Primary Clinical Imaging Parameters for Sequences 5 and 6 (Transverse PD- and $T_{2}$-Weighted FSE) ${ }^{a}$

\begin{tabular}{|c|c|}
\hline Patient position & Supine \\
\hline Scan type & 2-D FSE \\
\hline Imaging plane (orientation) & Transverse \\
\hline Variable bandwidth & Yes \\
\hline Central slice or volume center & Carotid bifurcation or stenosis \\
\hline Echo time $\left(T_{\mathrm{E}}\right)$ & $\begin{array}{l}\sim 7-12 \text { msec for sequence } 4, \\
\text { PD-weighted; } \sim 50-60 \mathrm{msec} \text { for } \\
\text { sequence } 5, T_{2} \text {-weighted }\end{array}$ \\
\hline Receiver bandwidth (RBW) & $\pm 31 \mathrm{kHz}$ or close \\
\hline Echo train length (ETL) & 8 \\
\hline Repeat time $\left(T_{\mathrm{R}}\right)$ & $\begin{array}{l}\text { 3-4 R-to-R intervals }{ }^{a}(\sim 2500-3000 \\
\text { msec) }\end{array}$ \\
\hline Fields of view $\left(\mathrm{FOV}_{x}, \mathrm{FOV}_{y}\right)$ & $160 \mathrm{~mm}, 120 \mathrm{~mm}$ \\
\hline Resolution $(\Delta x, \Delta y)$ & $0.63 \mathrm{~mm}, 0.63 \mathrm{~mm}$ \\
\hline Number of data points collected $\left(N_{\mathrm{x}}, N_{\mathrm{y}}\right)$ & 256,192 \\
\hline Display matrix $\left(D_{\mathrm{x}}, D_{\mathrm{y}}\right)$ & 512,512 \\
\hline Slice thickness $(\Delta z)$ & $2 \mathrm{~mm}$ \\
\hline Number of slices & $16-18$ \\
\hline Slice gap & $0 \mathrm{~mm}$ \\
\hline Number of acquisitions $\left(N_{\text {acq }}\right)$ & 2 \\
\hline Read direction & Left-right \\
\hline Slice locations & $\begin{array}{l}\text { Precise position relative to carotid } \\
\text { bifurcation }\end{array}$ \\
\hline Flow compensation & No \\
\hline ZIP 512 & Yes \\
\hline Saturation pulses & $\begin{array}{l}\text { Yes; superior and inferior } 50-60 \mathrm{~mm} \\
\text { slabs }\end{array}$ \\
\hline Fat suppression & Yes \\
\hline Scan time & $\sim 2.5-3 \mathrm{~min}$ \\
\hline
\end{tabular}

${ }^{a}$ Peripheral gating is required.

\section{Sequence 4: Transverse $T_{1}$-weighted black-blood fast-spin echo (FSE)}

23. Set up sequence parameters according to Table A1.4.6.

As in sequence 3, this sequence uses a DIR blood-suppression method. See annotations in step 15 for details. In the authors'standard protocol, sequence 3 is run without peripheral gating (using a custom-designed pulse sequence) to guarantee identical $T_{R}$, and, therefore, reproducible tissue contrast. Sequence 3 can potentially be run with gating, although a fast or slow heart rate may compromise the results. If a non-gated sequence is not available, one may also use a gating simulator programmed for the rate of 75 beats $/ \mathrm{min}$, i.e., $T_{R}=$ 800 msec. A particular problem in the setup of sequence 4 occurs on GE scanners with the standard commercial software, which does not permit black-blood acquisition with $T_{R}=1$ $R$-to- $R$ interval (only $2 R$-to- $R$ can be selected). The problem can be resolved by using an external gating simulator programmed for the appropriate heart rate, i.e., 150 beats $/ \mathrm{min}$, which results in $T_{R}=800$ msec for $2 R$-to- $R$ intervals. Alternatively, the sequence can be gated to the heart rate of the patient with $T_{R}=2 R$-to- $R$ intervals, but proper $T_{1}$-weighting may not be achieved. This should be taken into account when interpreting multi-contrast images. At the same time, this standard sequence provides effective blood suppression and can be used for lumen and wall identification.

MRI of

the Carotid

Artery for Atherosclerotic Plaques

A1.4.10 
Table A1.4.8 Alternate Imaging Parameters for Sequences 5 and 6 (Transverse PD- and $T_{2}$-Weighted FSE) with the Use of Multislice DIR Method ${ }^{a}$

\begin{tabular}{|c|c|}
\hline Patient position & Supine \\
\hline Scan type & 2-D FSE with DIR preparation \\
\hline Imaging plane (orientation) & Transverse \\
\hline Variable bandwidth & Yes \\
\hline Pulse sequence database (PSD) & "fse-m"a \\
\hline Central slice or volume center & Carotid bifurcation or stenosis \\
\hline Echo time $\left(T_{\mathrm{E}}\right)$ & $\begin{array}{l}\sim 7-12 \text { msec for sequence } 4, \\
\text { PD-weighted; } \sim 50-60 \mathrm{msec} \text { for } \\
\text { sequence } 5, T_{2} \text {-weighted }\end{array}$ \\
\hline Receiver bandwidth (RBW) & $\pm 31 \mathrm{kHz}$ or close \\
\hline Echo train length (ETL) & 12 \\
\hline Repeat time $\left(T_{\mathrm{R}}\right)$ & $2500 \mathrm{msec}(3000 \mathrm{msec})^{b}$ \\
\hline Inversion time $\left(T_{\mathrm{I}}\right)$ & $270 \mathrm{msec}(230 \mathrm{msec})^{b}$ \\
\hline Fields of view $\left(\mathrm{FOV}_{x}, \mathrm{FOV}_{y}\right)$ & $160 \mathrm{~mm}, 120 \mathrm{~mm}$ \\
\hline Resolution $(\Delta x, \Delta y)$ & $0.63 \mathrm{~mm}, 0.63 \mathrm{~mm}$ \\
\hline Number of data points collected $\left(N_{\mathrm{x}}, N_{\mathrm{y}}\right)$ & 256,192 \\
\hline Display matrix $\left(D_{\mathrm{x}}, D_{\mathrm{y}}\right)$ & 512,512 \\
\hline Slice thickness $(\Delta \mathrm{z})$ & $2 \mathrm{~mm}$ \\
\hline Number of slices & $8(12)^{b}$ \\
\hline Slice gap & $0 \mathrm{~mm}$ \\
\hline Number of acquisitions $\left(N_{\text {acq }}\right)$ & 2 \\
\hline Number of repetitions & 2 \\
\hline Read direction & Left-right \\
\hline Slice locations & $\begin{array}{l}\text { Precise position relative to carotid } \\
\text { bifurcation }\end{array}$ \\
\hline Flow compensation & No \\
\hline ZIP 512 & Yes \\
\hline Saturation pulses & No \\
\hline Fat suppression & Yes \\
\hline Slice series & Interleaved \\
\hline Scan time & $\sim 3 \mathrm{~min} / 8$ slices $(\sim 4 \mathrm{~min} / 12 \text { slices })^{b}$ \\
\hline \multicolumn{2}{|c|}{ 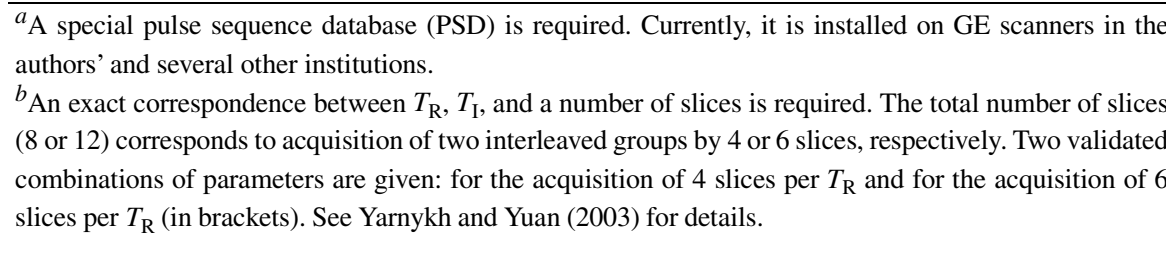 } \\
\hline
\end{tabular}

24. Prescribe a slice group (slab) at the specified distance from bifurcation (or exactly at the bifurcation) with the center of FOV between the left and right bifurcations (Fig. A1.4.2A). Use the coordinates determined in steps 19 and 21 to precisely position the slices.

Graphic displays for the prescription of scan geometry may not provide sufficient accuracy for this step. The authors recommend using exact numbers as coordinates for the slice group. Specifically, slices may be centered at the bifurcation or at the specified distance from the bifurcation based on the appearance of atherosclerotic plaque. This procedure enables the use of the bifurcation as a landmark for further comparison between images with different contrast weightings and, possibly, at different time periods in follow-up studies.

Intracranial Arterial Disease

A1.4.11 
Table A1.4.9 Primary Clinical Imaging Parameters for Sequence 7 (3-D TOF MRA)

\begin{tabular}{|c|c|}
\hline Patient position & Supine \\
\hline Scan type & 3-D spoiled gradient echo \\
\hline Imaging plane (orientation) & Transverse \\
\hline Variable bandwidth & Yes \\
\hline Central slice or volume center & Carotid bifurcation or stenosis \\
\hline Echo time $\left(T_{\mathrm{E}}\right)$ & $3.0-3.5 \mathrm{msec}$ \\
\hline Receiver bandwidth (RBW) & $\pm 12.5 \mathrm{kHz}$ or close \\
\hline Repeat time $\left(T_{\mathrm{R}}\right)$ & $20-25 \mathrm{msec}$ \\
\hline Flip angle (FA) & $25^{\circ}$ \\
\hline Fields of view $\left(\mathrm{FOV}_{x}, \mathrm{FOV}_{y}\right)$ & $160 \mathrm{~mm}, 120 \mathrm{~mm}$ \\
\hline Resolution $(\Delta x, \Delta y)$ & $0.63 \mathrm{~mm}, 0.63 \mathrm{~mm}$ \\
\hline Number of data points collected $\left(N_{\mathrm{x}}, N_{\mathrm{y}}\right)$ & 256,192 \\
\hline Display matrix $\left(D_{\mathrm{x}}, D_{\mathrm{y}}\right)$ & 512,512 \\
\hline Slice thickness $(\Delta z)$ & $2 \mathrm{~mm}$ \\
\hline Number of slices & 24 \\
\hline Slab thickness & $48 \mathrm{~mm}$ \\
\hline Number of slabs & 1 \\
\hline Slice gap & 0 \\
\hline Number of acquisitions $\left(N_{\text {acq }}\right)$ & 2 \\
\hline Read direction & Left-right \\
\hline Slice locations & $\begin{array}{l}\text { Precise position relative to carotid } \\
\text { bifurcation }\end{array}$ \\
\hline Flow compensation & Yes \\
\hline ZIP 512 & Yes \\
\hline ZIP 2 & Yes \\
\hline Saturation pulses & $\begin{array}{l}\text { Yes; superior } 50-80 \mathrm{~mm} \text { thick slab to } \\
\text { saturate veins }\end{array}$ \\
\hline Fat suppression & No \\
\hline Scan time & $\sim 3.5 \mathrm{~min}$ \\
\hline
\end{tabular}

Manual prescription of scan geometry may differ between manufacturers. It may require entering the position of the center of a slice group (Philips) or the position of the first (or last) slice (GE). In the latter case, the starting position (first slice) can be calculated using the position of the bifurcation (see step 21), number of slices, slice thickness, and slice gap. For example, if there are 16 contiguous slices with a thickness of $2 \mathrm{~mm}$ and it is necessary to center the slice group at the bifurcation, the starting inferior position will be $15 \mathrm{~mm}$ below the bifurcation. In this case, the eighth slice will be exactly at the bifurcation.

25. Instruct the patient to refrain from swallowing during the scan and begin the scan.

\section{Sequence 5: Transverse PD-weighted FSE}

26. Set up sequence parameters according to Table A1.4.7. Use proper echo time ( $\left.T_{\mathrm{E}}\right)$ for PD-weighted imaging from Table A1.4.7.

The sequence in Table A1.4.7 uses gated FSE acquisition with saturation of inflowing arterial and venous blood. This technique provides a fast scan with a large coverage but may suffer from residual flow artifacts. Improved flow suppression with little penalty of reduced coverage (or increased scan time) can be obtained using a new multislice DIR-FSE technique (Yarnykh and Yuan, 2003). If this pulse sequence is available, use Table A1.4.8 to set up parameters. 
Note that $P D$ - and $T_{2}$-weighted sequences 5 and 6 utilize identical parameters except for the echo time $\left(T_{E}\right)$. When setting up parameters according to Table A1.4.7 or Table A1.4.8, use proper $T_{E}$ as indicated for sequences 5 and 6.

27. Prescribe slices according to the above recommendations (see step 24).

28. Place inferior and superior saturation bands 2 to $3 \mathrm{~cm}$ from the edges of the slice group.

This step is not needed if a multislice DIR sequence is used (see Table A1.4.8).

29. Instruct the patient to refrain from swallowing during the scan and begin the scan.

Sequence 6: Transverse $T_{2}$-weighted FSE

30. Repeat steps 26 to 29 . Use proper echo time ( $\left.T_{\mathrm{E}}\right)$ for $T_{2}$-weighted imaging from Table A1.4.7.

Operators may copy the entire set of parameters from sequence 5 and change only $T_{E}$ according to Table A1.4.7. As with sequence 5, sequence 6 can be run using multislice DIR-FSE method. Refer to Table A1.4.8 in this case.

\section{Sequence 7: 3-D time-of-flight MRA}

31. Prior to scanning, ensure proper position of the imaged slab based on the above recommendations (see step 24). Run sequence 7 according to Table A1.4.9.

More details about 3-D TOF MRA of carotid arteries can be found in UNIT A1.3. The sequence parameters (Table A1.4.9) presented here are slightly different, as the primary goal of this sequence is to provide complementary information about the tissue composition of the atherosclerotic plaque rather than to visualize blood flow.

\section{COMMENTARY}

\section{Background Information}

Carotid atherosclerosis is thought to be one of the major contributors to stroke, which is among the leading causes of death and disability throughout the world. Traditionally, the degree of lumen stenosis is used as a marker for vulnerable plaques, which pose an increased risk for a thromboembolic event resulting in brain ischemia. However, two clinical trials (the North American Symptomatic Carotid Endarterectomy Trial and the Asymptomatic Carotid Atherosclerosis Study) demonstrated that lumen narrowing alone is a poor indicator of vulnerability, predicting only 1 out of 4 strokes in symptomatic patients and 1 out of 10 in asymptomatic patients. Many histological studies (for details see Yuan et al., 2001a) revealed the specific morphological factors of plaque instability. Some of these features are the erosion and rupture of the fibrous cap overlying the lipid core, the presence of a large necrotic core or intraplaque hemorrhage, and the appearance of calcium nodules on or near the lumen surface.

MRI is a noninvasive imaging technique, which can visualize the arterial lumen and provide detailed information about the arterial wall. Unlike the imaging modalities focused on the assessment of stenosis- $X$ ray and CT (computed tomography)-based angiography, and Doppler ultrasound-MRI has the unique potential to characterize various tissue components of atherosclerotic plaque. As a method for plaque characterization, MRI considerably outperforms alternative techniques - high-resolution CT and B-mode ultrasound - which have limited dynamic range for soft tissues (CT) or are prone to anatomic restrictions (B-mode ultrasound). A number of studies (Toussaint et al., 1996; Hatsukami et al., 2000; Yuan et al., 2001b) have shown that MRI is capable of identifying clinically relevant plaque constituents and morphological features in vivo, such as the lipid core, intraplaque hemorrhage, necrosis, calcified tissue, and ruptured fibrous cap. Due to the complexity of tissue components, multiple contrast weightings are needed for plaque characterization (Toussaint et al., 1996; Hatsukami et al., 2000; Yuan et al., 2001b). Good overall agreement between multi-contrast MRI and histology was documented for the classification and staging of atherosclerotic lesions (Cai et al., 2002). Additionally, MRI allows accurate measurements of
Intracranial Arterial Disease

A1.4.13

Supplement 11 
plaque size (Yuan et al., 1998; Kang et al., 2000), providing a means of monitoring the progression of atherosclerosis over time, as well as control of treatment.

High-resolution MRI of atherosclerotic plaques is a relatively new area, not commonly acknowledged as a routine diagnostic procedure. Recent successful applications of this method in relatively large groups of patients became possible due to the use of a specialized phased-array coil with improved SNR (Hayes et al., 1996; see also Fig. A1.4.1) and an optimized imaging protocol (Yuan et al., 2001a). Further improvement of image quality was achieved by using a new time-efficient blackblood imaging method, multislice DIR (Yarnykh and Yuan, 2003), although its availability is not critical for the Basic Protocol. In its current state, MRI of the atherosclerotic plaque requires the same scan time and efforts as many other MR protocols. Based on previous experience, the main goals of MRI in studies of carotid atherosclerosis are as follows: (1) detection and staging of the atherosclerotic disease; (2) monitoring of disease progression and effectiveness of therapy; and (3) detection of vulnerable atherosclerotic plaques, which are prone to disruption resulting in brain embolisms. The Basic Protocol provides comprehensive information for the assessment of all these aspects.

\section{Critical Parameters and Troubleshooting}

Image quality is critical for the identification of the tiny details of plaque structures. The following problems and artifacts are of major concern for high-resolution imaging of the carotid artery wall.

\section{Motion artifacts}

Swallowing is the major cause of motion artifacts in carotid imaging. It is especially important to instruct the patient to refrain from swallowing during each scan. For long singleslice DIR acquisition (sequences 3 and 4 ), some slices may be compromised due to motion, while others will be of good quality. In such situations, acquisition can be repeated for selected locations with little loss of overall examination time.

\section{Wrap-around artifacts}

The requirement of high resolution imposes limitations on the FOV size. The authors use a rectangular FOV for all transverse images to save scan time. Anterior and posterior parts of the neck may frequently appear outside the FOV and cause wrap-around artifacts (see, for example, Fig. A1.4.4). These are not critical unless they overlap with the carotid arteries. Wrap-around artifacts may create problems in patients with large necks. In this case, an increased $\mathrm{FOV}_{y}$ or a no-phase-wrap option (oversampling in the phase-encoding direction) can be used while keeping the same resolution, although scan time will be lengthened.

\section{Low SNR}

MR signal observed with a surface coil decreases with an increase of distance from the coil. If the area of interest (i.e., carotid arteries) appears far from the receiver panels of the coil (Fig. A1.4.1), image SNR may be insufficient. Another reason for a signal drop can be electromagnetic coupling between coil arrays, if receiver panels are improperly aligned. This may happen if panels are close to each other at the anterior surface of the neck and form an obtuse angle. Ideally, receiver panels should be parallel when placed at the lateral surfaces of the neck. If signal is low, try to readjust the coil position. This problem can be difficult to avoid in patients with a thick neck and deep carotid arteries. In such cases, SNR can be improved by a reasonable increase of the slice thickness (e.g., to 3 to $4 \mathrm{~mm}$ ).

\section{Incomplete flow suppression in black-blood imaging}

Effective blood suppression is critical for the evaluation of plaque components at the bloodwall interface. Sometimes, residual blood signal can be observed in the lumen, causing the lumen boundary to be obscured. Also, unsuppressed blood may result in the so-called plaque-mimicking artifact (Steinman et al., 1998). This artifact arises from recirculating blood and occurs mostly in the carotid bulb (Steinman et al., 1998). Effectiveness of flow suppression depends on the suppression technique used. The best results can be obtained using the double inversion-recovery (DIR) method (Edelman et al., 1991), which is utilized in sequences 3 and 4 . However, DIR is very time-consuming, since it requires single-slice acquisition. Due to scan-time limitations, DIR cannot be used in all dark-blood sequences (sequences 3 through 6). The inflow saturation method (used in sequences 5 and 6) is less effective, but it does not lengthen scan times. If present, plaque-mimicking artifacts on $\mathrm{PD} / T_{2^{-}}$ weighted images (sequences 5 and 6) can be easily recognized by comparing them with $T_{1^{-}}$ 
A

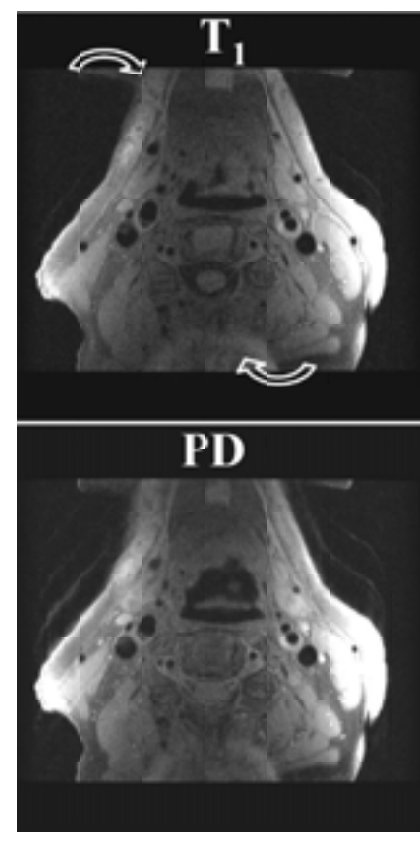

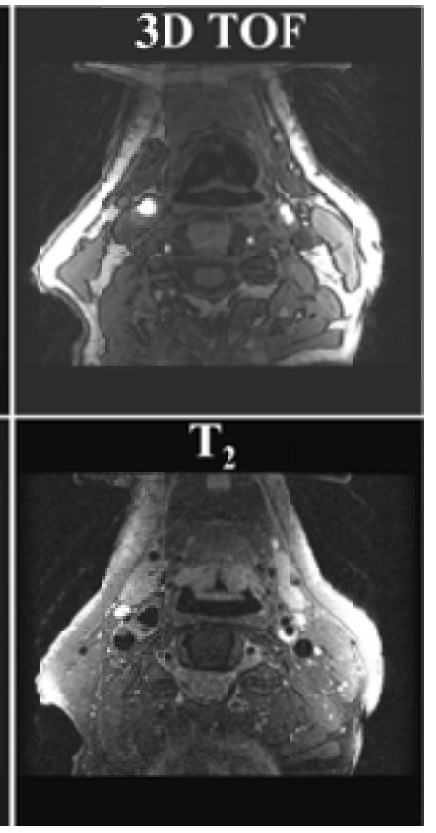

B

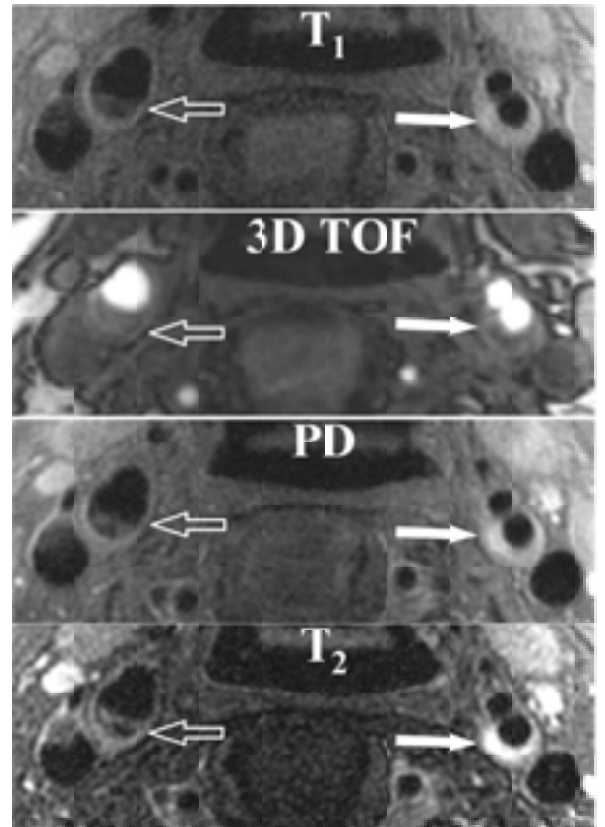

Figure A1.4.4 Transverse multi-contrast images (A) obtained from a patient with moderate atherosclerotic disease of the left carotid artery. Circular arrows indicate minor wrap-around artifacts. Magnified views of the carotid arteries (B) show atherosclerotic plaque overlaying the left internal carotid artery (solid arrows). This lesion contains a large fragment of loose fibrous matrix corresponding to a hyperintense area on the $T_{2}$-weighted image. In the right carotid bifurcation (open arrows), black-blood images display normal wall and a minor residual blood signal in the carotid bulb. This incompletely suppressed signal originates from recirculating blood and has minimal intensity on the $T_{1}$-weighted DIR image. Note the absence of flow enhancement in the right carotid bulb on the 3-D TOF image, which shows a tissue-like signal (open arrow). Without black-blood images, 3-D TOF MRA may result in misinterpretation of this region as plaque.

weighted images obtained by using DIR (sequence 4). Usually, these artifacts disappear or have dramatically reduced area and intensity on $T_{1}$-weighted DIR images.

Rarely, incomplete blood suppression may occur in DIR imaging (Fig. A1.4.4). This may happen if the time between DIR pulses and acquisition is insufficient for outflow of blood from an imaged slice, particularly, if blood recirculates or flows very slowly. If the sequence is used with cardiac gating, flow suppression may be compromised for very fast heart rates. A non-gated acquisition is preferable in this case (see also sequences 3 and 4). The critical parameters determining performance of DIR are the thickness of the inverted slice and the delay between a double-inversion pulse pair and a readout sequence. The delay is an analog of the inversion time $\left(T_{\mathrm{I}}\right)$ for inflowing blood. Usually, these parameters are automatically calculated by a scanner, but they can also be available for manual control (e.g., on Philips scanners). If a non-gated sequence is used, the optimal values of the delay are given in Tables A1.4.4 and A1.4.6. The thickness of an inverted slice should be 2 to 2.5 times larger than the thickness of an imaged slice. On Philips scanners, make sure that the thickness of an inverted slice is set to the minimal permitted value (currently $5 \mathrm{~mm}$ ).

An alternative solution for black-blood imaging with long $T_{\mathrm{R}}\left(\mathrm{PD}\right.$ and $T_{2}$-weighted) is based on recently developed multislice DIR methods (Parker et al., 2002; Song et al., 2002; Yarnykh and Yuan, 2003), which improve the time-efficiency of DIR and provide almost the same quality of blood suppression. One such multislice DIR technique was developed in the authors' institution (Yarnykh and Yuan, 2003), and it is used in the Basic Protocol (see Tables A1.4.5 and A1.4.8).

\section{Loss of flow enhancement in 3-D TOF MRA}

Sequence 7 (3-D TOF MRA) may suffer from specific artifacts resulting in a reduced signal in the vessel lumen. Due to the absence
Intracranial Arterial Disease

A1.4.15

Supplement 11 
of flow enhancement, such areas can be erroneously interpreted as tissue (parts of the plaque) or thrombosis (Fig. A1.4.4). Common reasons for these artifacts are due to the saturation of slow flow and flow-dependent dephasing. These issues are discussed in detail in UNITS A1.3 \& B7.3. In the Basic Protocol, 3-D TOF is used as an additional contrast weighting ( $T_{1}$-weighted gradient-echo contrast), which is interpreted in conjunction with black-blood images. If complementary high-quality blackblood images are available, signal-loss artifacts in the lumen area on TOF images can be reliably distinguished from the plaque (Fig. A1.4.4).

\section{Anticipated Results}

Examination with the Basic Protocol provides comprehensive characterization of the atherosclerotic lesion (Figs. A1.4.3 and A1.4.4). Usually, both lumen and plaque are clearly visible on all dark-blood images. Oblique sagittal black-blood MRA depicts the distribution of the plaque along the artery and lumen narrowing in a stenotic segment (Fig. A1.4.3). Transverse images obtained with DIR blood suppression allow excellent contrast between wall and lumen (Fig. A1.4.4). Brightblood MRA (3-D TOF) is generally unable to depict the lumen-wall boundary as conspicuously as the black-blood images (Fig. A1.4.4). However, TOF images provide helpful complementary contrast features. Slice-by-slice comparison of images with four basic contrast weightings $\left(T_{1}, T_{2}, \mathrm{PD}\right.$, and gradient-echo TOF) offers identification of basic plaque components, such as fibrous tissue, lipid core, necrosis, calcifications, and hemorrhages. The typical signal features of plaque tissues can be found in a review by Yuan et al. (2001a). The main plaque constituent is fibrous tissue with signal characteristics similar to muscle. Early plaques are usually composed of fibrous tissue and display uniform signal intensity. During evolution, plaques acquire lipids and calcifications, and also may undergo necrosis or incidental changes due to hemorrhages and ruptures. Advanced plaques frequently show areas of variable signal. Specifically, hyperintensity on $T_{1}$-weighted images may be associated with either an intraplaque hemorrhage or the lipid core; both are destabilizing factors making the plaque prone to rupture. TOF images may help to distinguish a recent hemorrhage and the lipid core, since a recent hemorrhage (in contrast to the lipid core) frequently shows increased signal on TOF images (Yuan et al., 2001b). At the same time, signal characteristics of a hemor- rhage are stage-dependent. Hyperintensity on $T_{2^{-}}$and PD-weighted images in combination with iso-/hypointense signal on $T_{1}$-weighted images indicate a so-called loose matrix, which is a less integrated fibrous tissue with an elevated liquid phase. This may be a result of an old hemorrhage and necrosis. Calcified regions can be identified as dark areas in all images. Bright-blood TOF images are especially helpful to assign calcificates close to the lumen surface (juxtalumenal calcium) or to identify a ruptured fibrous cap (Hatsukami et al., 2000). Plaque rupture is an important clinical condition, which is associated with neurological symptoms (stroke and transient ischemic attack). By comparing black- and bright-blood images, plaque rupture can be identified as a dark/bright tear originating at the lumen boundary.

Finally, black-blood images can be used for quantitative morphologic analysis, such as lumen and wall area and volume measurements (Yuan et al., 1998; Kang, 2000). Precise morphometry of the atherosclerotic plaque is now becoming an important tool for studies of disease progression and treatment.

\section{Acknowledgments}

The authors would like to thank Dr. Baocheng Chu for helpful comments; Drs. Jeffrey Maki and Gregory J. Wilson for assistance in implementing this protocol for a Philips scanner; and Marina S. Ferguson for reviewing the manuscript.

\section{Literature Cited}

Cai, J.M., Hatsukami, T.S., Ferguson, M.S., Small, R., Polissar, N.L., and Yuan, C. 2002. Classification of human carotid atherosclerotic lesions with in vivo multicontrast magnetic resonance imaging. Circulation 106:1368-1373.

Edelman, R.R., Chien, D., and Kim, D. 1991. Fast selective black blood MR imaging. Radiology 181:655-660.

Hatsukami, T.S., Ross, R., Polissar, N.L., and Yuan, C. 2000. Identification of fibrous cap thickness and cap rupture in human atherosclerotic carotid plaque in vivo with high resolution magnetic resonance imaging. Circulation 102:959-964.

Hayes, C.E., Mathis, C.M., and Yuan, C. 1996. Surface coil phased arrays for high-resolution imaging of the carotid arteries. J. Magn. Reson. Imaging 6:109-112.

Kang, X.J., Polissar, N.L., Han, C., Lin, E., and Yuan, C. 2000. Analysis of the measurement precision of arterial lumen and wall areas using high resolution magnetic resonance imaging. Magn. Reson. Med. 44:968-972. the Carotid

Artery for

Atherosclerotic

Plaques

A1.4.16 
Parker, D.L., Goodrich, K.C., Masiker, M., Tsuruda, J.S., and Katzman, G.L. 2002. Improved efficiency in double-inversion fast spin-echo imaging. Magn. Reson. Med. 47:1017-1021.

Shellock, F.G. 2001. Pocket Guide to MR Procedures and Metallic Objects. Lippincott-Raven, Philadelphia.

Song, H.K., Wright, A.C., Wolf, R.L., and Wehrli, F.W. 2002. Multislice double inversion pulse sequence for efficient black-blood MRI. Magn. Reson. Med. 47:616-620.

Steinman, D.A. and Rutt, B.K. 1998. On the nature and reduction of plaque-mimicking flow artifacts in black blood MRI of the carotid bifurcation. Magn. Reson. Med. 39:635-641.

Toussaint, J.F., LaMuraglia, G.M., Southern, J.F., Fuster, V., and Kantor, H.L. 1996. Magnetic resonance images lipid, fibrous, calcified, hemorhagic, and thrombotic components of human atherosclerosis in vivo. Circulation 94:932938.

Yarnykh, V.L. and Yuan, C. 2003. Multislice double inversion-recovery black-blood imaging with simultaneous slice reinversion. J. Magn. Reson. Imaging 17:478-483.

Yuan, C., Beach, K.W., Smith, L.H., and Hatsukami, T.S. 1998. Measurement of atherosclerotic carotid plaque size in vivo using high resolution magnetic resonance imaging. Circulation 98:2666-2671.

Yuan, C., Mitsumori, L.M., Beach, K.W., and Maravilla, K.R. 2001a. Carotid atherosclerotic plaque: Noninvasive MR characterization and identification of vulnerable lesions. Radiology 221:285-299.

Yuan, C., Mitsumori, L.M., Ferguson, M.S., Polissar, N.L., Echelard, D., Ortiz, G., Small, R., Davies, J.W., Kerwin, W.S., and Hatsukami, T.S. 2001b. In vivo accuracy of multispectral magnetic resonance imaging for identifying lipidrich necrotic cores and intraplaque hemorrhage in advanced human carotid plaques. Circulation 104:2051-2056.
Key References

Shellock, F.G. 2001. Magnetic Resonance Procedures: Health Effects and Safety. CRC Press, Boca Raton, Fla.

Covers a number of important patient management issues related to MR imaging, including recommended safety procedures, a list of metallic implants that have been tested for MR compatibility, and a list of other sources on MR safety.

Yarnykh and Yuan, 2003. See above.

Describes technical principles and demonstrates advantages of a multislice DIR method, which is preferable for performing sequences 3,5 , and 6 in the Basic Protocol. The method produces excellent results and it is easy for implementation.

Yuan et al., 2001a. See above.

Comprehensive review of clinical and technical aspects of carotid plaque MRI.

\section{Internet Resources}

http://vil.rad.washington.edu

Web site of the Vascular Imaging Lab (University of Washington) contains example images and useful references on MRI of the carotid atherosclerotic plaque.

http://www.mrisafety.com

Covers a number of important patient management issues related to MR imaging, including recommended safety procedures, a list of metallic implants that have been tested for MR compatibility, and a list of other sources on MR safety.

Contributed by Vasily L. Yarnykh and Chun Yuan

University of Washington

Seattle, Washington
Intracranial Arterial Disease

A1.4.17 